\title{
Medical Professionals Have Higher Risk for Colorectal Adenoma But Lower Risks for Reflux Esophagitis and Atrophic Gastritis: A Propensity Score-Based Analysis
}

Chen Qiao

Shandong University Qilu Hospital

Min Zhang

Shandong University Qilu Hospital

Zhen Li

Shandong University Qilu Hospital

Xiu-Li Zuo ( $\boldsymbol{\nabla}$ zuoxiuli@sdu.edu.cn )

Shandong University Qilu Hospital

Research article

Keywords: gastrointestinal disease, physician, colorectal adenoma, reflux esophagitis, atrophic gastritis

Posted Date: August 27th, 2020

DOI: https://doi.org/10.21203/rs.3.rs-61804/v1

License: (c) (1) This work is licensed under a Creative Commons Attribution 4.0 International License.

Read Full License 


\section{Abstract}

Background: Previous studies had indicated that medical professionals' lifestyles were not as healthy as recommended. Whether these lifestyles lead to higher incidences of gastrointestinal diseases is unknown.

Objectives: To compare the incidences of gastrointestinal diseases between medical professionals and general populations.

Methods: Subjects who underwent endoscopy examination for physical examination in Qilu Hospital from January 2017 to September 2019, were retrospectively reviewed. Propensity score based analysis was used to generate two age- and sex-matched groups, Medical Professional Group and General Population Group. The positive rates of gastrointestinal disorders in two groups, were analyzed by Pearson's Chi-square test. Subgroup analysis within Medical Professional Group was performed to identify risk factors for medical staffs.

Results: 1534 records were enrolled, 791 for gastroscope and 743 for colonoscope. Among upper gastrointestinal diseases, the detection rates of atrophic gastritis and reflux esophagitis showed significant differences, which were 19.8\% in Medical Professional Group and 25.9\% in General Population Group ( $p=.04$ ) for atrophic gastritis, $4.0 \%$ in Medical Professional Group and $12.6 \%$ in General Population Group $(p<0.001)$ for reflux esophagitis. Among the subjects who received colonoscopy, the adenoma detection rate of the Medical Professional Group (17.5\%) was statistically distinguished from the General Population Group (11.5\%) $(p=.02)$.

Conclusion: In a provincial comprehensive hospital in China, medical Professional Group has significantly higher risk for colorectal adenoma, but lower risks for atrophic gastritis and reflux esophagitis.

\section{Introduction}

Recently, there is increasing recognition that the health conditions of medical professionals require more attention. ${ }^{1}$ Many medical professionals lost sight of their own personal health while they confront the stresses of increased workload to deliver daily care and services to patients. In this context, enhancing their personal health practice becomes very essential. It does not only improve their overall work performance but also helps them to better guide patients. ${ }^{2-4}$ It has been reported that physicians' personal health practice is one of the strongest predictors of whether they are capable to offer helpful lifestyle advice to patients. ${ }^{5}$ Indeed, one study in Canada confirmed a positive association between preventive health practices of physicians and that of patients. ${ }^{6}$

In fact, lifestyles of physicians have attracted more and more attentions. An international review of tobacco smoking in medical professionals has shown a steady decline of it in most developed countries, but doctors in newly developing and several other developed countries were still holding on smoking at 
high rates. ${ }^{7}$ Other studies have also shown smoking ranges in different countries from $2 \%$ in the US to $49 \%$ in Greece. ${ }^{7}$ Another focus on physicians' lifestyles is alcohol using. In a cross-sectional survey in the UK, many clinical staffs took more alcohol than recommendation. ${ }^{8}$ A nationwide study in Denmark found the risky alcohol use rates in physicians were from 17.2-20.3\%. ${ }^{9}$ Another national cross-sectional study in Ireland compared the alcohol consuming rates between physicians and general populations, and found that physicians were more likely to consume alcohol than general population but less likely to engage in binge drinking. ${ }^{10}$ Apart from these problems, physical activities and burnout of physicians have also been well studied. Researches have shown that many medical staffs do inadequate physical exercises. ${ }^{8,10}$ And burnout is common among medical workers, especially young medical staffs. ${ }^{11}$ They often live in reverse life around the clock. ${ }^{12}$ Long working hours coupled with psychological stress are also barriers to a healthy life. 8,13

In a word, the medical professional's lifestyles do not always meet the requirements, even some of them are unhealthy. These lifestyles are exactly the potential risk factors for gastrointestinal diseases. For example, both physical activity and psychological stress are associated with a variety of digestive diseases. ${ }^{14-19}$

Do physicians have a higher risk for gastrointestinal diseases? Until now, statistics are lacking and the answer is unclear to us. So, we conducted this retrospective study to compare the incidence of gastrointestinal diseases diagnosed by endoscopy between medical professionals and general populations. Our study aims to provide new evidences in the epidemiology of gastrointestinal diseases of medical professionals, and trying to give some reasonable suggestions based on these data.

\section{Materials And Methods}

\section{Subjects}

This is a single center, retrospective, propensity score-matched analyzed study. Subjects who underwent gastroscopy or colonoscopy for physical examinations in Qilu Hospital of Shandong University, China, from January 2017 to September 2019, were retrospectively analyzed. Exclusion criteria included: (1) age less than 50 years old or more than 79 years old, (2) a history of gastric cancer or colonrectal cancer, (3) a history of gastrointestinal surgical or endoscopic operations, (4) unfinished endoscopy procedures due to poor coordination, poor bowel or gastric preparations or high operating difficulties (Uncompleted procedures caused by newly diagnosed severe diseases were not ruled out.), and (5) records with incomplete information.

All the included subjects were divided into two groups, Medical Professional Group and General Population Group. Medical professionals referred to all permanent staffs in Qilu Hospital, including doctors, assistant personnel and administrative staffs. General population referred to all those who visited the Health Screening Center of Qilu Hospital for gastrointestinal examinations or who visited 
gastrointestinal clinic for healthy screening endoscopic examinations. Subjects in the two groups were age- and sex-matched.

\section{Endoscopic procedures}

Informed consents for gastroscopy or colonoscopy were granted from all the subjects before the procedures. For gastroscopy, intubation of duodenal descending part was required and described in the reports with words and pictures. And for colonoscopy, the bowel preparation agents used were all polyethylene glycol-based regimens. Polyethylene glycol was Polyethylene Glycol Electrolytes Powder (Wanhe Pharmaceutical Co. Ltd., Shenzhen, China). Depth of insertion and cecal intubation were required fields for report completion. Cecal intubation was documented by the endoscopist using landmark descriptions including "ileocecal valve" and "appendiceal orifice" with an associated photograph. The endoscopic procedures and diagnosis were completed by experienced endoscopists with more than 5 years experiences in endoscopy performance. All the pathological diagnoses of biopsy samples were also made by experienced pathologists.

\section{Primary outcomes and secondary outcomes}

The endoscopic and pathological diagnosis were reviewed and recorded. The primary outcomes of the study were the positive rates of gastrointestinal disorders, including reflux esophagitis, gastric polyp, peptic ulcer, $\mathrm{H}$. pylori infection, atrophy gastritis, gastric cancer, colorectal polyp, adenoma and cancer, colorectal ulcer, melanosis coli and colonic diverticula. H. pylori infection was ensured by histopathology or rapid urease test (RUT) of mucosa from corpus or antrum.

The secondary outcomes were the subgroup analysis within the Medical Professional Group, which included the incidences of gastrointestinal diseases between males and females, clinicians and nonclinicians, surgeons and non-surgeons, and among different ages.

\section{Statistical analysis}

Statistical analysis was performed using SPSS 23.0. Continuous variables were expressed as means (standard deviation, SD) and compared with Student's t test; categorical variables were expressed as percentages and were compared with the Pearson's Chi-square test.

Propensity scores (PSs) were estimated via a multiple logistic regression model to balance the two groups with respect to age and sex. ${ }^{20}$ One-to-one nearest-neighbor matching was performed between Medical Professional Group and General Population Group with a caliper set at 0.1 . The resulting scorematched groups were used in subsequent analysis. The age- and sex-balance were verified by Student's $t$ test and Pearson's Chi-square test separately. Upon obtaining satisfactory balance between the two groups, the primary outcomes, the positive rates of gastrointestinal disorders, were analyzed using Pearson's Chi-square test. To further investigate the differences among medical professionals, subgroupanalysis was performed within the Medical Professional Group. Pearson's Chi-square test was also used to assess incidences between subgroups divided by genders, ages and specialties. The significant level was set at $p<0.05$. 


\section{Results}

A total of 3181 records were reviewed, 1597 for gastroscopy and 1562 for colonoscopy after dropping duplicate records. Finally, 414 records were excluded from gastroscopy records, of which 397 records due to ages, 14 due to surgical or endoscopic operation histories, and 3 due to incomplete information. As for colonoscopy, 412 records were excluded, of which 395 for younger or older ages, 6 for surgical or endoscopic operation histories, 9 for unfinished colonoscopy and 2 for incomplete information. After oneto-one propensity score matching, 1534 records were enrolled for further analysis, 791 for gastroscopy and 743 for colonoscopy. Exactly, 893 subjects were enrolled in the study and 641 of the subjects received both gastroscopy and colonoscopy at the same visits. The flowchart of reviewing and enrolling the participants was shown in Fig. 1. The participants were age- and sex- matched between Medical Professional Group and General Population Group. The clinical characteristics were shown in Additional Table 1. No significant differences were found between the two groups with respect to ages and genders.

Among upper gastrointestinal diseases, the detection rates of reflux esophagitis, gastric polyp, peptic ulcer, $\mathrm{H}$. pylori infection, atrophic gastritis and the gastric cancer were analyzed. The detection rates of atrophic gastritis and reflux esophagitis are significantly lower in Medical Professional Group than General Population Group, $19.8 \%$ versus $25.9 \%(p=.04)$ for atrophic gastritis and $4.0 \%$ versus $12.6 \%$ ( $p<$ 0.001) for reflux esophagitis (Fig. 2, Additional Table 2). The detection rates of colorectal polyp, adenoma, colorectal cancer, colorectal ulcer, melanosis coli and colonic diverticula among subjects who received colonoscopy examinations were shown in Fig. 3. The colorectal adenoma detection rate of the Medical Professional Group (17.5\%) was statistically distinguished from the General Population Group $(11.5 \%)(p=.02)$ (Fig. 3 and Additional Table 3$)$.

Subgroup analysis within Medical Professional Group indicated a lower level of gastric polyp detection rate in male $(14.5 \%)$ than female $(28.7 \%)(p=.002)$. But male physicians had a significantly higher risk (58.9\%) for colorectal polyp compared with females (39.4\%) $(p=.001)$ (Table 1 and Table 2). For the comparison between surgeons and non-surgeons, the positive rate of reflux esophagitis and gastric polyp were $8.6 \%$ versus $2.9 \%(p=.03)$ and $14.3 \%$ versus $25.6 \%(p=.04)$ respectively (Table 3$)$. Incidences of lower gastrointestinal diseases were similar in surgeons and non-surgeons (Additional Table 4). No significant differences were found in gastrointestinal diseases when comparing the clinical staff and non-clinical staff ( Additional Table 5 and Additional Table 6). 
Table 1

The positive rates of upper gastrointestinal diseases in males and females within Medical Professional Group.

\begin{tabular}{|llll|}
\hline Positive Rate $(\%)$ & Male $(\mathrm{n}=1 \mathbf{1 3 8})$ & Female $(\mathrm{n}=\mathbf{2 4 0})$ & $\boldsymbol{p}$ value \\
\hline Reflux esophagitis & 5.1 & 3.3 & .40 \\
\hline H. pylori & 24.6 & 28.7 & .39 \\
\hline Atrophic gastritis & 22.5 & 18.3 & .33 \\
Gastric cancer & 0.7 & 0.4 & $>.99$ \\
\hline Peptic ulcer & 4.3 & 4.2 & .93 \\
Gastric polyps & 14.5 & 28.7 & $.002^{\text {** }}$ \\
\hline
\end{tabular}

Table 2

The positive rates of lower gastrointestinal diseases in males and females within Medical Professional Group.

\begin{tabular}{|llll|}
\hline Positive Rate $(\%)$ & Male $(\mathrm{n}=112)$ & Female $(\mathrm{n}=\mathbf{2 1 3})$ & $\mathbf{p \text { value }}$ \\
\hline Colorectal polyp & 58.9 & 39.4 & $.001^{\text {** }}$ \\
\hline Colorectal adenoma & 23.2 & 14.6 & .05 \\
\hline Colorectal cancer & 0.9 & 0.9 & $>.99$ \\
\hline Colorectal ulcer & - & - & - \\
\hline Melanosis coli & 1.8 & 3.3 & .72 \\
\hline Colonic diverticula & 3.6 & 2.8 & .74 \\
\hline
\end{tabular}

Table 3

The positive rates of upper gastrointestinal diseases in surgeons and nonsurgeons within Medical Professional Group.

\begin{tabular}{|llll|}
\hline Positive Rate $(\%)$ & Surgeon $(n=70)$ & Non-surgeon $(n=308)$ & $p$ value \\
\hline Reflux esophagitis & 8.6 & 2.9 & $.04^{*}$ \\
\hline H. pylori & 32.9 & 26.0 & .24 \\
\hline Atrophic gastritis & 20.0 & 19.8 & .97 \\
Gastric cancer & 1.4 & 0.3 & .33 \\
\hline Peptic ulcer & 1.4 & 4.9 & .32 \\
Gastric polyp & 14.3 & 25.6 & $.04^{*}$ \\
\hline
\end{tabular}




\section{Discussion}

Results show that positive rate of colorectal adenoma in medical professionals is higher than in age- and sex-matched general population. The positive rates of atrophic gastritis and reflux esophagitis in medical professionals are lower than general population. Subgroup analysis within the medical professionals indicates positive rates of gastric cancer and colorectal cancer get higher with age (Additional Table 7 and Additional Table 8). It is suggested that medical professionals' health consciousness for gastrointestinal diseases should be promoted. And regular screening endoscopic examinations, especially colonoscopy, are recommended.

The underline mechanisms for these results are not exactly known. However, previous studies have provided us some potential risk factors based on lifestyles, such as fewer physical activities, burnout and psychological stress. ${ }^{8,10-13}$ Their beliefs, varied lifestyle may cause co-effects to the differences. Understanding the mechanism helps for potential screening and preventive measures for gastrointestinal diseases in medical professionals, which will potentially influence the healthy condition of the communities. One underlying reason may be the health beliefs and practices of physicians. Reflux esophagitis and chronic gastritis often present with symptoms like heartburn, pain, belching and abdominal distension, which can be easily recognized and relieved by simple prescriptions by medical professionals. Yet colorectal adenomas tend to be asymptomatic abnormalities, which basically show no warning signal. So, even reflux esophagitis, chronic gastritis and colorectal adenoma share similar risk factors in clinical settings, the positive rates of the diseases are diverse in two groups.

Gender-based subgroup analysis within the medical professionals indicates higher gastric polyp positive rate but lower colorectal polyp positive rate in female, which is consistent with those of the general population. Whereas surgeons tend to a higher reflux esophagitis positive rate and a lower gastric polyp positive rate than non-surgeons. These may due to different working environment and stress. Unexpectedly, the comparison results between clinical and non-clinical staff for gastrointestinal diseases is insignificant, which may serve as supporting proof of comparable concerns for non-clinical staff in the hospital.

There are several limitations of this study. First, this is a retrospective study in one single center. But propensity score-based analysis was used to reduce bias. Second, the subgroup analysis within physicians did not adjust for some important potential confounding factors, like age, gender and diets. Because the subject number in subgroups analysis was not sufficient to be fully matched or adjusted. Third, the lifestyles and related risk factors of subjects were not investigated in this study. Prospective studies are needed to provide more exact evidence and guidance for physicians' healthcare.

\section{Conclusion}

In conclusion, this study provided us the most recent statistical evidence for the heathy condition of gastrointestinal tract in medical professionals in a provincial comprehensive hospital, which was a wake- 
up call for all doctors. This study was based on but also an extension of previous articles which analyzed the characteristics of physician's lifestyles. The lifestyle changes and endoscopy screening for medical professionals should be valued, especially for colonoscopy examination.

\section{Abbreviations}

RUT: rapid urease test

\section{Declaration}

\section{Ethics approval and consent to participate}

The study was approved by Medical Ethics Committee of Qilu Hospital of Shandong University. The consent of patients was waived by the hospital's ethical committee.

\section{Consent for publication}

Not applicable.

\section{Availability of data and materials}

The datasets used and analyzed during the current study are available from the corresponding author on reasonable request.

\section{Competing interests}

The authors declare that they have no competing interests.

\section{Funding}

This study was supported by the Primary Research and Development Plan of Shandong Province under Grant 2016GSF201134 and Primary Research and Development Plan of Shandong Province under Grant $2017 G S F 218069$.

\section{Authors' contributions}

Chen Qiao, Zhen Li and Xiuli Zuo designed the study. Chen Qiao and Min Zhang collected the data. Chen Qiao and Zhen Li analyzed the data and interpreted the results. Chen Qiao drafted the manuscript. Zhen Li and Xiuli Zuo revised the manuscript. Chen Qiao, Min Zhang, Zhen Li and Xiuli Zuo interpreted the data and reviewed the manuscript. All authors read and approved the final manuscript.

\section{Acknowledgement}

The authors would like to express their gratitude to the staff of the endoscopy department and out-patient department at the Qilu Hospital for their assistance. 


\section{References}

1. Taub S, Morin K, Goldrich MS, Ray P, Benjamin R. Physician health and wellness. Occup Med (Lond). 2006; 56(2):77-82

2. Rose SA, Poynter PS, Anderson JW, Noar SM, Conigliaro J. Physician weight loss advice and patient weight loss behavior change: a literature review and meta-analysis of survey data. Int $\mathrm{J}$ Obes (Lond). 2013; 37(1):118-128

3. Stead LF, Buitrago D, Preciado N, Sanchez G, Hartmann-Boyce J, Lancaster T. Physician advice for smoking cessation. Cochrane Database Syst Rev. 2013; 31:5

4. Grandes G, Sanchez A, Sanchez-Pinilla RO, et al. Effectiveness of physical activity advice and prescription by physicians in routine primary care: a cluster randomized trial. Arch Intern Med. 2009; 169(7):694-701

5. Oberg EB, Frank E. Physicians' health practices strongly influence patient health practices. J R Coll Physicians Edinb. 2009; 39(4):290-291

6. Frank E, Dresner $Y$, Shani M, Vinker S. The association between physicians' and patients' preventive health practices. CMAJ. 2013; 14;185(8):649-653

7. Smith DR, Leggat PA. An international review of tobacco smoking in the medical profession: 19742004. BMC Public Health. 2007; 20; 7:115

8. Mittal TK, Cleghorn CL, Cade JE, et al. A cross-sectional survey of cardiovascular health and lifestyle habits of hospital staff in the UK: Do we look after ourselves? Eur J Prev Cardiol. 2018; 25(5):543550

9. Sørensen JK, Pedersen AF, Bruun NH, Christensen B, Vedsted P. Alcohol and drug use among Danish physicians. A nationwide cross-sectional study in 2014. Dan Med J. 2015; 62(9)

10. O' Keeffe A, Hayes B, Prihodova L. "Do as we say, not as we do?" the lifestyle behaviours of hospital doctors working in Ireland: a national cross-sectional study. BMC Public Health. 2019; 11;19(1):179

11. Romani M, Ashkar K. Burnout among physicians. Libyan J Med. 2014; 17; 9:23556

12. Mota MC, De-Souza DA, Rossato LT, et al. Dietary Patterns, Metabolic Markers and Subjective Sleep Measures in Resident Physicians. Chronobiol Int. 2013; 30(8):1032-1041

13. Hayes B, Prihodova L, Walsh G, Doyle F, Doherty S. What's up doc? A national cross-sectional study of psychological wellbeing of hospital doctors in Ireland. BMJ Open. 2017; 16;7(10): e018023

14. Cheng Y, Macera CA, Davis DR, Blair SN. Does physical activity reduce the risk of developing peptic ulcers? Br J Sports Med. 2000; 34(2):116-121

15. Brown JC, Winters-Stone K, Lee A, Schmitz KH. Cancer, Physical Activity, and Exercise. Compr Physiol. 2012; 2(4): 2775-2809

16. Mawdsley JE, Rampton DS. Psychological stress in IBD: new insights into pathogenic and therapeutic implications. Gut. 2005 Oct;54(10):1481-1491 
17. Jansson C, Wallander MA, Johansson S, Johnsen R, Hveem K. Stressful psychosocial factors and symptoms of gastroesophageal reflux disease: a population-based study in Norway. Scand J Gastroenterol. 2010;45(1):21-29

18. Levenstein S, Rosenstock S, Jacobsen RK, Jorgensen T. Psychological stress increases risk for peptic ulcer, regardless of Helicobacter pylori infection or use of nonsteroidal anti-inflammatory drugs. Clin Gastroenterol Hepatol. 2015;13(3):498-506.e1

19. Qin HY, Cheng CW, Tang XD, Bian ZX. Impact of psychological stress on irritable bowel syndrome. World J Gastroenterol. 2014; 21;20(39):14126-14131

20. Yang HJ, Guo Z, Yang YT, et al. Blood neutrophil-lymphocyte ratio predicts survival after hepatectomy for hepatocellular carcinoma: A propensity score-based analysis. World J Gastroenterol. 2016;22(21):5088-5095.

\section{Figures}

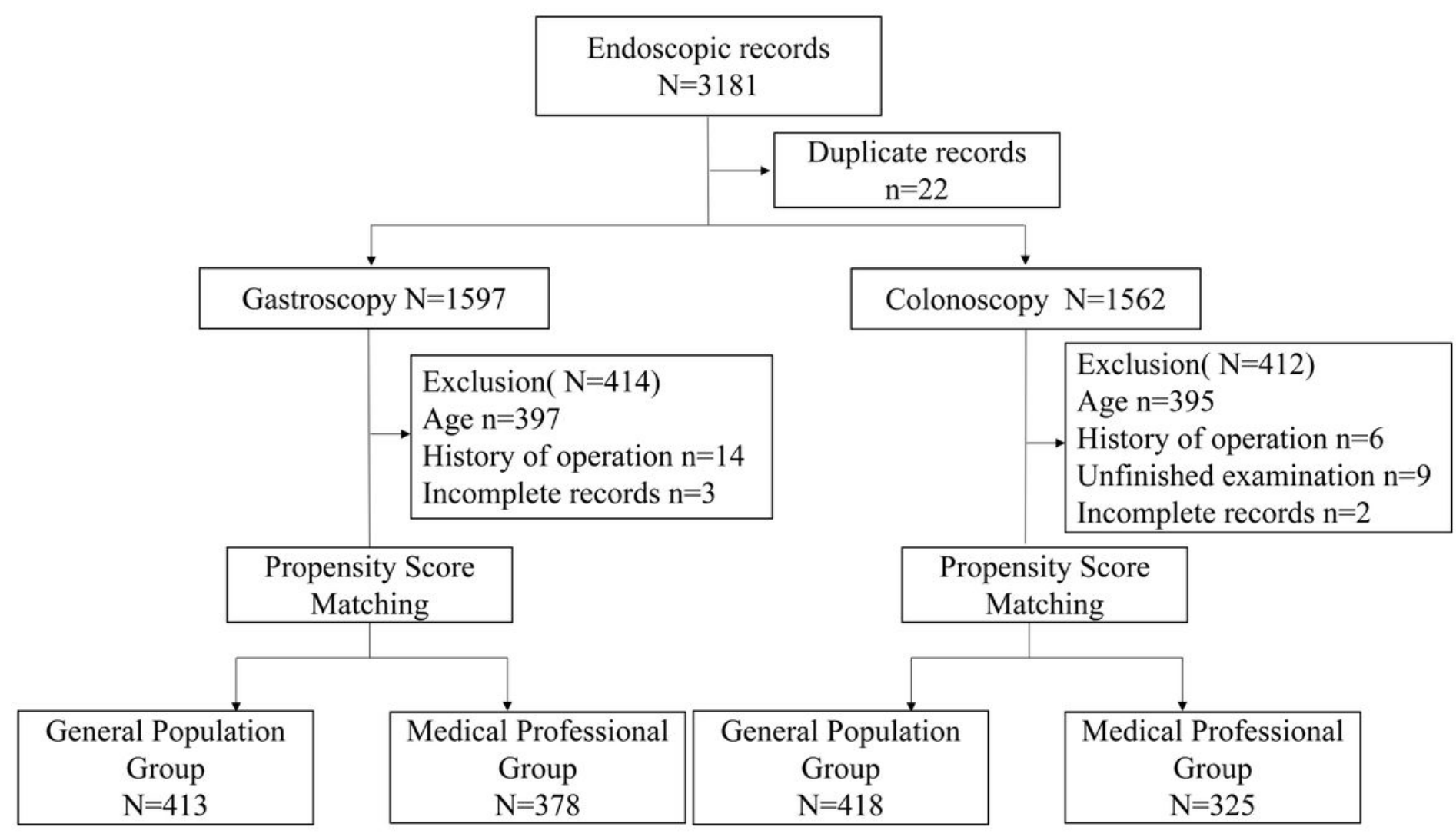

\section{Figure 1}

The flowchart of reviewing and enrolling the participants 


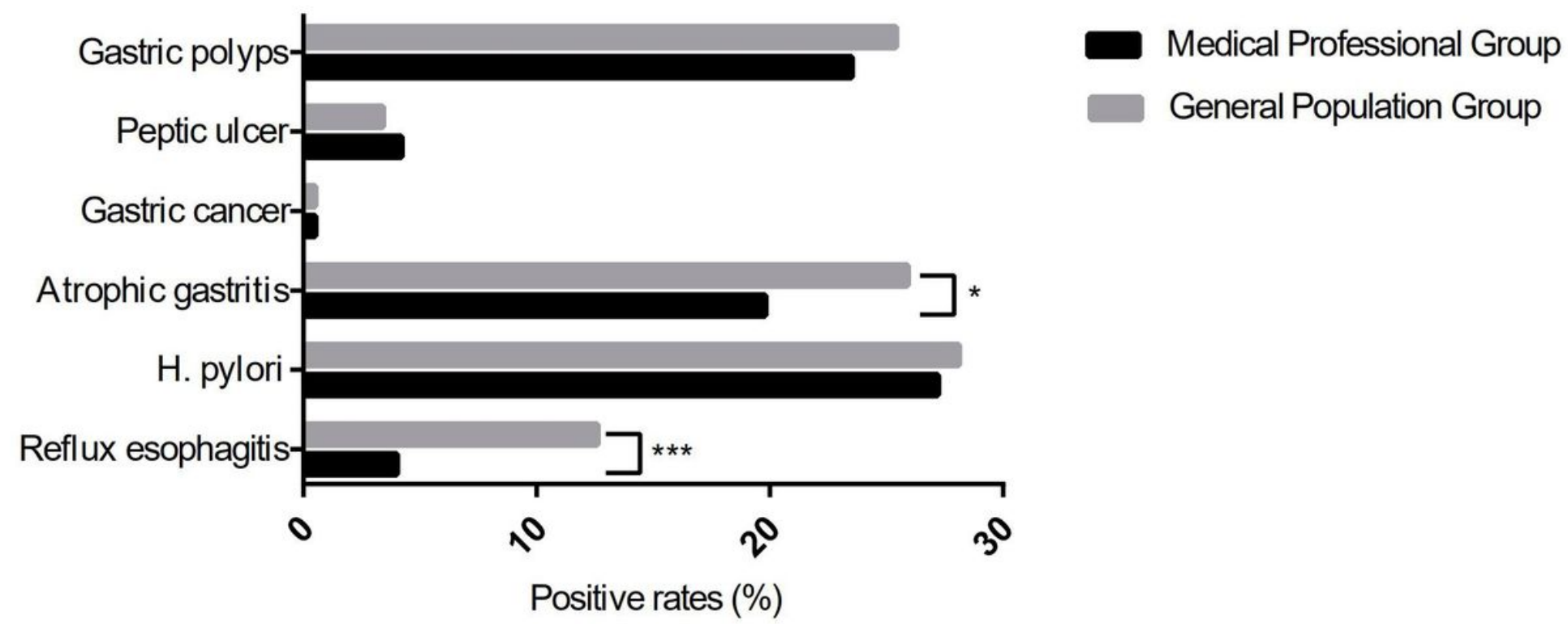

Figure 2

The disease spectrum of upper gastrointestinal tract in Medical Professional Group and General Population Group

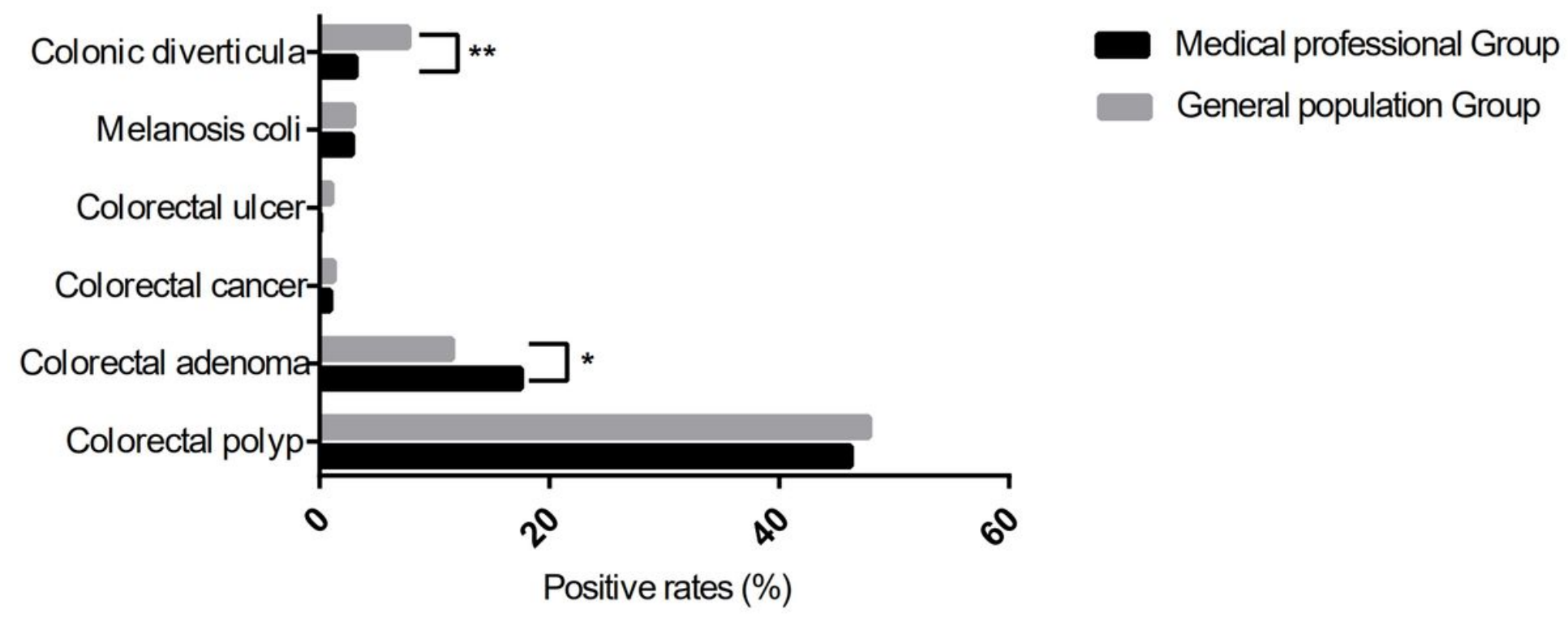

Figure 3

The disease spectrum of lower gastrointestinal tract in Medical Professional Group and General Population Group

\section{Supplementary Files}


This is a list of supplementary files associated with this preprint. Click to download.

- AdditionalTables.docx 